\title{
Endovascular Treatment for Carotid Blowout Syndrome with Acute Hemorrhage in Head and Neck Cancers: A Report of Two Cases
}

Pietro Modugno ${ }^{1}$, Enrico M. Centritto ${ }^{1}$, Veronica Picone ${ }^{1}$, Francesco Fraticelli $^{2}$, Antonio Pierro ${ }^{3}$, Savino Cilla ${ }^{4 *}$, Francesco Deodato $^{5}$ Carlo M. De Filippo ${ }^{6}$ and Vincenzo Valentini ${ }^{7}$

${ }^{1}$ Vascular Surgery Unit, Gemelli Molise Hospital, Catholic University of Sacred Heart, Campobasso, Italy ${ }^{2}$ Intensive Care and Resuscitation Department, Gemelli Molise Hospital, Catholic University of Sacred Heart, Campobasso, Italy ${ }^{3}$ Radiology Department, Gemelli Molise Hospital, Catholic University of Sacred Heart, Campobasso, Italy ${ }^{4}$ Medical Physics Unit, Gemelli Molise Hospital, Catholic University of Sacred Heart, Campobasso, Italy ${ }^{5}$ Radiation Oncology Unit, Gemelli Molise Hospital, Catholic University of Sacred Heart, Campobasso, Italy ${ }^{6}$ Cardiac Surgery Department, Gemelli Molise Hospital, Catholic University of Sacred Heart, Campobasso, Italy ${ }^{7}$ Radiation Oncology Department, Foundazione Policlinico Universitario Gemelli, Catholic University of Sacred Heart, Roma, Italy ${ }^{\star}$ Corresponding author: Savino Cilla, Medical Physics Unit, Gemelli Molise Hospital, Campobasso, Italy, Tel: 0039-0874-312360; E-mail: savino.cilla@gemellimolise.it Received: May 20, 2020; Accepted: June 05, 2020; Published: June 08, 2020

\begin{abstract}
We present two cases of carotid blowout syndrome with a hemorrhagic shock treated with an endovascular approach. In the first case, the treatment was performed by a selective embolization, while in the other case, both embolization and positioning of a covered stent was performed in order to avoid bleeding of external carotid artery. These endovascular treatments allowed the resolution of bleeding and the survival of the patients. Unfortunately, both patients died six months after the procedure for the evolution of their neoplastic disease. The endovascular treatment of carotid blowout syndrome represents a valid therapeutic option to stop carotid bleeding, but it does not change the prognosis of the patients.
\end{abstract}

Keywords: Carotid blowout, Covered stent, Hemorrhage

\section{Introduction}

The incidence of significant hemorrhage in patients with Head and Neck cancer is approximately 6-14\%, while terminal hemorrhages occur in approximately $3-12 \%$ of cases [1,2]. Head and neck cancer is the eighth most common cancer worldwide, and account for approximately $3 \%$ of all malignant tumors [3]. These are a broad category including diverse types originating from different anatomical structures like craniofacial bones, soft tissues, salivary glands, skin, and mucosal membranes with squamous cell carcinoma, which is the most common histological type (90\%) [4]. The 5-years survival rate for head and neck cancers is around 60\% [3]. Although chemotherapy and radiotherapy have improved the life expectancy of patients with head and neck cancers, they have also increased the risk of hemorrhage up to 7.6 times [4-6]. These cancers are the most common cancers associated with hemorrhage, with an incidence rate ranging from 0.5 to $10 \%[2,6,7]$. Carotid blowout syndrome is a rare, life threatening complication in patient with advanced head and neck cancer [8]. It refers to the rupture of the carotid artery or its extracranial branches, usually secondary to tumor encasement or invasion. It can occur after good response to radical treatment, in which there is loss of tissue bulk surrounding and supporting an artery due to previous tumor invasion. Untreated carotid blowout syndrome is associated with mortality rates up to $60 \%$ and neurological morbidity of $40 \%$ [9]. Risk factors for carotid blowout syndrome in patients with head and neck cancer include post-radiotherapy necrosis, recurrent tumors, pharyngo-cutaneous fistulas, poor nutrition, diabetes mellitus and prolonged corticosteroid use [10]. When acute bleeding occurs from carotid blowout, treatment options are surgical ligation, endovascular embolization, or reconstruction with covered stents [11]. Surgery in a previously irradiated area may be challenging, and emergent operative ligation can be associated with higher rate of major morbidity [9].

\section{Cases Report}

We reported two cases of carotid blowout syndrome with acute hemorrhage in patients with head and neck cancer.

\section{Case 1}

A 49-year old man was diagnosed in October 2017 with squamous carcinoma of the tongue. He had previous medical history and pots actively. After biopsy he was treated with neoadjuvant chemotherapy. The patient received 3 cycles of chemotherapy with DCF without complication. CT imaging showed partial response. The case was discussed at a multi-disciplinary tumor board and concomitant radiotherapy and chemotherapy with platinum was initiated. After 
the treatment the patient refused examinations. Seven month later, the patient was hospitalized due to an aggravation of the clinical conditions. CT imaging documented a local recurrence of cancer. During the hospitalization a respiratory disorder was presented. Tracheostomy was urgently performed. Unfortunately, a massive hemorrhage appeared with hemodynamic instability and hemorrhagic shock. The patient after intensive care, the patient underwent the endovascular treatment urgent with embolization of the external carotid artery and its branches (Figure 1). There were no central neurological complications (Stroke ischemic or TIAs) and after 12 hours the bleeding ceased with restoration of good haemodynamic compensation. There were no episodes of re-bleeding during the hospital stay. The patient was discharged and transferred to Hospice. The patient died after 6 months due to the progression of the neoplastic disease which led him to acute respiratory failure.

\section{Case 2}

A 66-year-old patient underwent hemimandibulectomy with resection of the pterygoid and masseter muscles in July 2017. A histological examination showed a squamous carcinoma. After surgery, six cycles of chemotherapy comprising Cisplatin, 5-FU and Cetuximab was administered without complication. After 3 months a whole-Body 18F-FDG PET/CT showed lymph node progression. Second-line chemotherapy with the Carboplatin-Taxol was initiated without complication. After 3 cycles of chemotherapy, platinumbased radio-chemotherapy was started in December 2018. In January 2019 he showed rapid clinical progression of the disease and was admitted to our Department. During the hospitalization a massive hemorrhage appeared without hemodynamic instability. Therefore the patient underwent urgent CT of the neck which showed the presence of a pseudoaneurysm of the external carotid artery but a bleb of the common carotid artery immediately before the carotid bifurcation caused by neoplastic tissue sleeve around the bifurcation caused by neoplastic tissue sleeve around the bifurcation (Figure 2). Therefore it was necessary to undergo in urgency the patient not only to embolization of the pseudoaneurysm of the external carotid artery with controlled release spirals (Axium EV-3) but also to reconstruct the common carotid artery by positioning a stent covered Viabahn (Gore) between the common and internal carotid arteries in order to avoid the rupture of the vessel downstream of the pseudoaneurysm by the sleeve of neoplastic tissue present around (Figure 3). There were no

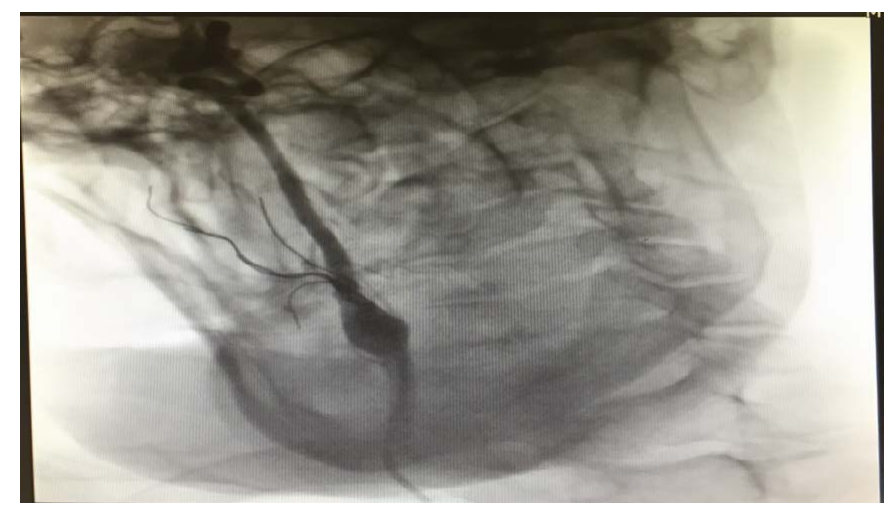

Figure 1: Endovascular treatment with embolization ECA and its branches. central neurological complications (Stroke ischemic or TIAs) and the bleeding stopped immediately. The patient was immediately treated with dual anti-aggregation therapy to avoid intrastent thrombosis and there were not episodes of re-bleeding during the hospital stay. The patient was discharged and suspended chemo-radiation treatment

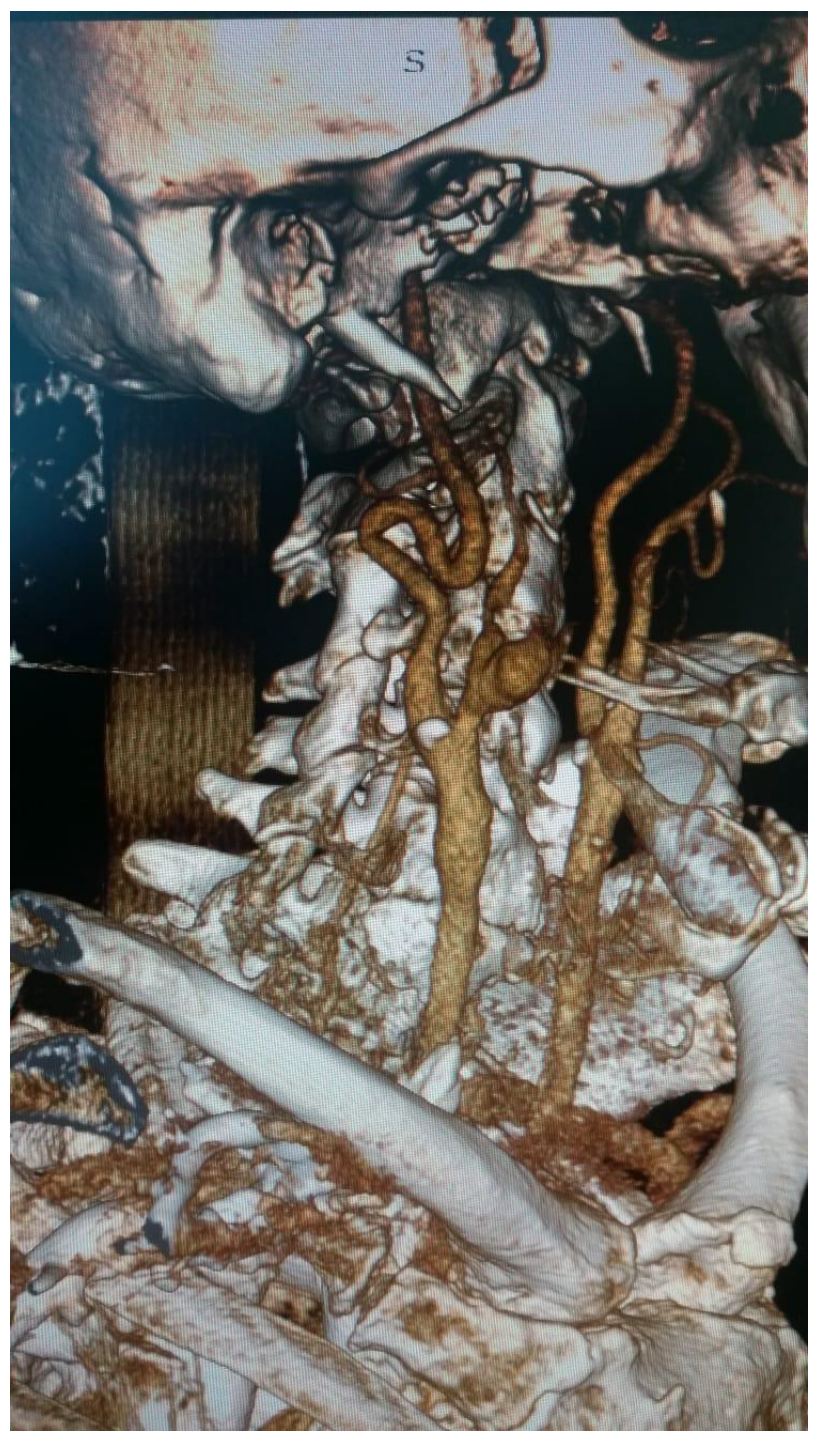

Figure 2: Angio-CT vessels neck showing pseudoaneurysm ECA e neoplastic tissue around carotid bifurcation.

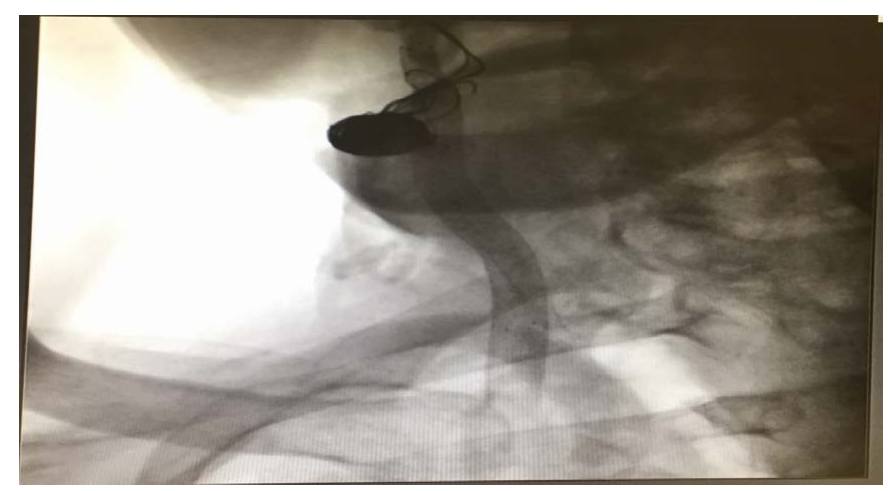

Figure 3: Endovascular treatment with embolization ECA and its branches e covered stent graft between ICA and CCA. 
and sent for supportive therapy. After 6 months the patient is still alive even though in progression with the neoplastic disease and did not present new episodes of bleeding or signs of capped stent infection.

\section{Discussion}

Carotid Blowout Syndrome (CBS) is an uncommon complication of the advanced head and neck cancer. With continuing advancement and availability of endovascular techniques, an endovascular approach to be treatment of carotid blowout syndrome has been demonstrated to be safe and has potential to control acute bleeding events and improve quality of life. The incidence has been estimated to be between $2 \%$ and $5 \%$ in patients receiving intervention for head and neck cancer, up to $10 \%$ in those receiving repeated courses of radiation. The morbidity of CBS has been described, with perioperative mortality rate as high as $30 \%$ and a perioperative stroke rate as high as $15 \%$, but remain highly variable owing to the rarity of the condition $[12,13]$. Both embolization and stent grafts have been used in the management acute or impending carotid blowout. The most common locations or tumor were in the oral cavity and larynx and tho most common bleeding origin was the common carotid artery [14]. Both endovascular treatment were successful technically for immediate bleeding cessation in those with acute hemorrhage, but with a stroke rate of around $11 \%$. The risk of procedural stroke was lowest with embolizaion for CBS in the external carotid artery $1.7 \%$, followed by stent graft of internal carotid artery/ common carotid artery (ICA/CCA) $2.5 \%$ and then embolization of ICA/CCA $10.3 \%$ with post-treatment fatal re-bleeding occurring only $3.4 \%$ [11]. This compares favorably to the natural history of untreated carotid blowout syndrome, with associated risk of neurological morbidity up to $40 \%$ and mortality rate up to $60 \%$ [9].

Embolization of the ICA/CCa was generally preceded by the balloon test occlusion and stent graft was more likely to be performed in the presence of contralateral carotid artery occlusion, intolerance of balloon occlusion testing or angiographic evidence of an incomplete circle Willis [15]. Notably, despite the balloon test occlusion, the procedural stroke rate was higher after embolization of the ICA/CCA (10.4\%) compared to the stent graft (2.5\%). The rebreeding rate after stent graft was higher (31.9\%) compared with embolization (9.1\%), which may relate to the use of dual antiplatelet agents after stent grafts, which are typically not required after embolization . Importantly, $94 \%$ of patients treated for CBS involving the ICA/ACC did not experience fatal re-bleeding, with no difference between embolisation and stent grafts [15]. Chang et al. Included 96 patients with CBS of which had lesion in the CCA, ICA, or bifurcation: 38 patients were treated with embolization after passing a balloon occlusion test, and 18 patients underwent stent graft. Similarly, procedural stroke rates were similar (embolization $10.5 \%$; stent graft $11.1 \%$ ) and significantly higher rates of rebleeding in group stent graft $38.9 \%$ versus embolization $13.2 \%$.

For embolization of CBS arising from branches of the External Carotid Artery, the procedural stroke rate was $<2 \%$. Although there were no fatal re-bleeds, another episode of bleeding did occur in $30 \%$ of the patients. This is similar to the largest case series by Chang et al. [16] in which $35 \%$ of patients had rebleeding [16]. The reason for rebleeding is not clear, and was not extractable from the literature. Potential reasons for rebleeding may relate to lack of identification of a target vessel, or retrograde flow from the rich collateral network of the external carotid artery when proximal occlusion of a target vessel is performed. Moreover, a new lesion of the ECA crunch may rise or the tumor bed could rebleed as the tumor continues to recruit neovascularity [16,17]. Moreover these were a higher rate of delayed stroke or TIA after stent grafts compared to embolization of the ICA/CCA. Although this could relate to delayed stent thrombosis, the majority (two-third) of stent thrombosis were asymptomatic. In addition, delayed stroke could relate to use post stent graft antiplatelet agents. The most common regimen was clopidogrel and aspirin foe 1-3 months, followed by life-long aspirin use. The antiplatelet regimens used are generally tailored to the clinical presentation, balancing the individual risk of rebleeding and ischemic stroke [11].

In the two cases treated, we first tried to stop the massive bleeding. In the first case where there was a hemorrhagic shock we immediately did the embolization of the external carotid artery not only of the main trunk but also of the branches of division, using spirals. In fact there was no neurological complication of ischemic type, nor new bleeding. in the second case there was no hemodynamic instability linked to bleeding which required to stop the bleeding immediately. Thus it was possible to perform a CT scan of the neck vessels which allowed identifying not only the origin of the bleeding but also the extent of the neoplastic disease. In fact, the simple embolization of the external carotid artery and its own pseudoaneurysm due to neoplastic infiltration of the wall would have temporarily solved the bleeding. In fact, the presence at CT of a neoplastic tissue sleeve around the carotid bifurcation with initial contour alteration would soon lead to a new bleeding. Therefore we changed surgical strategy proceeding both to the embolization of the pseudoanerysm of the external carotid artery and of the main trunk and to a reconstruction endovascular with the Stent graft covered Viabahn (gore) $7 \times 10 \mathrm{~mm}$, positioned between the internal carotid artery and the common carotid artery, covering the outer external carotid artery ostium and the neoplastic tissue sleeve around the carotid bifurcation. there was no neurological complication of ischemic type, nor new bleeding Therefore this work exposes a small and limited experience to only two cases but, however reliable, there were no complications and the patients survived the massive bleeding of Carotid Blowout Syndrome. Important is the planning of endovascular strategy.

\section{Conclusion}

The endovascular approach appears to be, in our opinion, the gold standard for the treatment of bleeding from neoplasms, perhaps relapses, of head and neck after tumor removal with associated lymphadenectomy, or chemotherapy and subsequent radiotherapy. In fact, both the embolization of the external carotid artery or ICA/CCA, and the placement of a covered stent have proved and are reliable for the immediate control of the acute hemorrhage in progress. Complications can possibly be controlled if proper operative intervention is planned based on the location of the source of the bleeding and the extent of the neoplastic disease. For this reason it is fundamental to perform a CT scan of the neck vessels, if the patient's haemodynamic conditions allow it, in order to evaluate the type of endovascular intervention to be carried out to not only stop bleeding but reduce the risk of perioperative complications. 


\section{References}

1. Ubogagu E, Harris DG (2012) Guideline for the management of terminal Hemorrhage in palliative care patients with advanced cancer discharged home for end-to-life care. BMJ Support Palliat Care 2: 294-300. [Crossref]

2. Harris DG, Noble SI (2009) Management of terminal hemorrhage in patients with advanced cancer; a systematic literature review. J Pain Symptom Manag 38: 913-927. [Crossref]

3. Jemal A, Siegel R, Xu J, Ward E (2010) Cancer Statistics, 2010. CA Cancer J Clin 60: 277-300. [Crossref]

4. Pai SI, WestraWH (2009) Molecular Pathology of head and neck cancer: implications for diagnosis, prognosis, and treatment. Ann Rev. Pathol 4: 49-70. [Crossref]

5. Ringash J (2015) Survivorship and quality of life in head and neck cancer. J Clin Oncol 33: 3322-3327. [Crossref]

6. Self EM, Bunpous J, Ziegler C, Wilson L, Potts K (2013) Risk factors for hemorrahage after chemoradiation for oropahryngeal squamous cell carcinoma. JAMA Otolaryngol Head Neck Surg 139: 356-361. [Crossref]

7. Hessén Soderman AC, Ericson E, Hemlin C, Hemlin C, Hultcrantz E, et al. (2011) Reduced risk of primary postoperartive hemorrhage after tonsil surgery in Sweden: results from The National Tonsil Surgery Register in Swedwe covering more tha 10 years and 54696 operations. Laryngoscope 121: 2322-2326. [Crossref]

8. Upile T, Triaridis S, Kirland P, Archer D, Searle A, et al. (2005) The management of carotid artery rupture. Eur Arch Otorhinolaryngol 262: 555-560. [Crossref]

9. Chaloupka JC, Putman CM, Citardi MJ, Ross DA, Sasaki CT (1996) Endovascular Therapy for the carotid blowout syndrome in head and neck surgical patientsdiagnostic and managerial considerations. AJNR Am J Neuroradiol 17: 843-852. [Crossref]
10. McDonald MW, Moore MG, Johnstone PA (2012) Risk of carotid blowout after reirradiation of the head and neck: a systematic review. Int J Radiat Oncol Bio Phys 82: 1083-1089. [Crossref]

11. Wong DJY, Donaldson C, Lai LT, Coleman A, Giddings C, et al. (2017) Safety and effectiveness of endovascular embolization or stent-graft reconstruction for treatment of acute carotid blowout syndrome in patients with head and neck cancer: case series and systematic review of observational studies. Head \& Neck 40: 846-854. [Crossref]

12. Cohen J, Rad I (2004) Contemporary Management of carotid blowout. Curr Opin Otolaryngol Head Neck Surg 12: 110-115. [Crossref]

13. Morrisey DD, Andersen PE, Nesbit GM, Barnwell SL, Everts EC, et al. (1997) Endovascular management of hemorrhage in patients with head and neck cancer. Arch Otolaryngol Head Neck Surg 123: 15-9. [Crossref]

14. Liang NL, Guedes BD, Duvvuri U, Singh MJ, Chaer RA, et al. (2016) Outcomes of interventions for carotid blowout syndromein patients with head and neck cancer. J Vasc Surg 63: 1525-1530. [Crossref]

15. Lesley WS, Chaloupka JC, Weigele JB, Mangla S, Dogar MA (2003) Preliminary experience with endovascular reconstruction of the management of carotid blowout syndrome. AJNR AmJ Neuroradiol 24: 975-981. [Crossref]

16. Chang FC, Luo CB, Lirng JF, Lin CJ, Lee HJ, et al. (2015) Endovascular management of post-irradiated carotid blowout syndrome. PLoS One 10: e0139821. [Crossref]

17. Behzad SF, Lee DH, Khalil A, Abrol S, Flores L (2014) Carotid Blowout Syndrome: Endovascular management of acute Hemorrhage with Tapering Overlapped Covered Stents. Ann Vasc Surg 28: 1934.e7-11. [Crossref]

\section{Citation:}

Pietro Modugno, Enrico M. Centritto, Veronica Picone, Francesco Fraticelli, Antonio Pierro, et al. (2020) Endovascular Treatment for Carotid Blowout Syndrome with Acute Hemorrhage in Head and Neck Cancers: A Report of Two Cases. J Cardiol Clin Pract Volume 3(2): 1-4. 